\title{
Systematic treatment of displacements, strains and electric fields in density-functional perturbation theory
}

\author{
Xifan $\mathrm{Wu}^{1}$, David Vanderbilt ${ }^{1}$, D.R. Hamann ${ }^{2}$ \\ ${ }^{1}$ Department of Physics and Astronomy, Rutgers University, Piscataway, New Jersey 08854-8019, USA \\ ${ }^{2}$ Bell Laboratories, Lucent Technologies, Murray Hill, NJ 08974
}

(January 28, 2005)

\begin{abstract}
The methods of density-functional perturbation theory may be used to calculate various physical response properties of insulating crystals including elastic, dielectric, Born charge, and piezoelectric tensors. These and other important tensors may be defined as second derivatives of the total energy with respect to atomic-displacement, electric-field, or strain perturbations, or as mixed derivatives with respect to two of these perturbations. The resulting tensor quantities tend to be coupled in complex ways in polar crystals, giving rise to a variety of variant definitions. For example, it is generally necessary to distinguish between elastic tensors defined under different electrostatic boundary conditions, and between dielectric tensors defined under different elastic boundary conditions. Here, we describe an approach for computing all of these various response tensors in a unified and systematic fashion. Applications are presented for two materials, wurtzite $\mathrm{ZnO}$ and rhombohedral $\mathrm{BaTiO}_{3}$, at zero temperature.
\end{abstract}

77.65.-j, 62.20.Dc, 77.22.Ch, 77.65.Bn, 77.84.-s, 71.15.Mb

\section{INTRODUCTION}

The methods of density-functional theory $(\mathrm{DFT})^{1}$ and density-functional perturbation theory (DFPT) $)^{2,3}$ have been shown to give a successful description of the dielectric and piezoelectric properties of a wide range of materials in which electronic correlations are not too strong. ${ }^{4,5}$ Many properties of interest can be computed directly from DFT using finite differences - for example, elastic constants computed from the stress arising from a small applied strain, or dynamical effective charges computed from polarizations ${ }^{6}$ arising from small sublattice displacements. On the other hand, the use of DFPT methods is becoming increasingly popular because it can be used to compute such response properties directly, without the need for multiple ground-state calculations, thus providing the desired response properties in a more automated, systematic, and reliable fashion.

As a result, improved DFPT capabilities have been implemented in recent years in several of the computer code packages commonly used by the computational electronic-structure community. ${ }^{7-9}$ This development has been most thorough in the case of the opensource ABINIT computer package, ${ }^{7}$ in which the capability for handling strain perturbations ${ }^{10}$ has recently been added to the previous implementation of atomicdisplacement and electric-field perturbations. This development opens the prospect for a systematic treatment of three kinds of perturbations in insulating crystals on an equal footing: periodicity-preserving atomic displacements (i.e., zone-center phonons), homogeneous electric fields, and homogeneous strains. These three degrees of freedom are often strongly coupled, especially in polar materials used in modern ferroelectric, piezoelectric, and dielectric applications.
In this work, we show that such a systematic approach is now not only practical, but especially powerful. That is, we show that computing the full set of six elementary (or "bare") response tensors (force-constant, dielectric, elastic-constant, Born-charge, internal-strain, and piezoelectric tensors) associated with these three kinds of perturbations provides an extremely valuable database that can subsequently be used to compute a wide variety of relevant physical properties. Among these, for example, are the physical dielectric, elastic, and piezoelectric tensors (in which atomic displacements have been taken into account), elastic compliances, free-stress dielectric tensors, fixed-electric-displacement elastic tensors, alternative piezoelectric tensors, and electromechanical coupling constants. The ability to access this wide range of secondary properties becomes possible only after the full set elementary response tensors has been systematically computed.

The rest of this paper is organized as follows. In Sec. II, we present the formal development, defining the various elementary response tensors and showing how other response tensors of interest can be derived from these. Then in Secs. III-IV we apply our approach to wurtzite $\mathrm{ZnO}$ and rhombohedral $\mathrm{BaTiO}_{3}$ as two paradigmatic example systems. We first briefly describe the practical details of the calculations in Sec. III, and then present the results for the ground-state properties, elementary response properties, and derived response properties, in Sec. IV. We conclude with a summary in Sec. V. A careful formulation of the theory for the case in which strains and electric fields are simultaneously present is deferred to the Appendix. 


\section{FORMALISM}

\section{A. Elementary response tensors}

Consider an insulating crystal with $N$ atoms per unit cell. We choose a reference state in which the lattice vectors are $\mathbf{a}_{1}, \mathbf{a}_{2}$, and $\mathbf{a}_{3}$, the cell volume is $\Omega_{0}$, and the atomic coordinates are $R_{m}^{(0)}$. Here $m$ is a composite label (atom and displacement direction) running over $1, \ldots, 3 N$, and we assume that this structure is the equilibrium one in vanishing macroscopic electric field.

We consider three kinds of perturbations applied to such a crystal: (i) displacements $u_{m}$ of the atoms away from their equilibrium positions, (ii) homogeneous strains $\eta_{j}$ where $j=\{1 \ldots 6\}$ in Voigt notation, and (iii) homogeneous electric fields $\mathcal{E}_{\alpha}$ where $\alpha=\{x, y, z\}$ are Cartesian directions. We restrict our discussion to atomic displacements that preserve the primitive-cell periodicity, i.e., to degrees of freedom corresponding to zone-center phonon modes only. Also, we will restrict ourselves entirely to zero-temperature properties.

The corresponding responses that are conjugate to these three perturbations are (i) forces $F_{m}$, (ii) stresses $\sigma_{j}$, and (iii) polarizations $P_{\alpha}$. From these, one can construct the response functions of primary interest: "diagonal" responses $K_{m n}=d F_{m} / d u_{n}$ (force-constant matrix), $\chi_{\alpha \beta}=d P_{\alpha} / d \mathcal{E}_{\beta}$ (dielectric susceptibility), and $C_{j k}=d \sigma_{j} / d \eta_{k}$ (elastic constants), and "off-diagonal" response tensors $Z_{m \alpha}=d P_{\alpha} / d u_{m}$ (Born effective charge), $\Lambda_{m j}=d F_{m} / d \eta_{j}$ (internal strain), and $e_{\alpha j}=d P_{\alpha} / d \eta_{j}$ (piezoelectric response). However, in order to define these quantities carefully, it is important to clarify the constraints or boundary conditions that apply to each definition. For example, the elastic constants $C_{j k}$ may be defined allowing or not allowing internal atomic displacements ("relaxed-ion" or "frozen-ion"), or under conditions of fixed electric $(\mathcal{E})$ or displacement $(\mathbf{D})$ field.

We take the approach here of systematically defining all response properties as appropriate second derivatives of the energy $E$ per unit volume with respect to the perturbations. To be more precise, in the presence of strains we define $E$ as the energy per undeformed unit cell volume $\Omega_{0}$, while in the presence of electric fields $E$ is modified to become an electric enthalpy ${ }^{11}$ by adding a term proportional to $-\mathbf{P} \cdot \mathcal{E}$, where $\mathbf{P}$ is the electric polarization. ${ }^{6}$ (While a direct treatment of finite $\mathcal{E}$-fields is now possible, ${ }^{12,13}$ only infinitesimal $\mathcal{E}$-fields need to be considered here.) In general, we define $E$ as

$$
E(u, \mathcal{E}, \eta)=\frac{1}{\Omega_{0}}\left[E_{\text {cell }}^{(0)}-\Omega \mathcal{E} \cdot \mathbf{P}\right]
$$

where $E_{\text {cell }}^{(0)}$ is the usual zero-field Kohn-Sham energy per cell ${ }^{14}$ of the occupied Bloch functions and $\Omega$ is the deformed cell volume. However, when strains and electric fields are simultaneously present, care is needed in the interpretation of Eq. (1); this is explained in the Appendix, where a more precise formulation is given in the form of Eq. (A11), which supersedes Eq. (1). In short, the difficulty is connected with the distinction between "proper" and "improper" piezoelectric constants; ${ }^{15}$ we should like our formulation to lead to the former and not the latter. The factor of $\Omega / \Omega_{0}$ has been inserted in the last term of Eq. (1) towards this purpose, but this is not sufficient by itself. In addition, Eq. (1) should be rewritten in terms of "natural variables" $u, \mathcal{E}^{\prime}$, and $\eta$, where $\mathcal{E}$ has been replaced by a reduced electric field $\mathcal{E}^{\prime}$ that is defined in Eq. (A5). When partial derivatives are taken with respect to these natural variables, one automatically obtains the "proper" piezoelectric tensors. Indeed, as explained in the Appendix, all appearances of $\mathcal{E}$ should be replaced by $\mathcal{E}^{\prime}$, with a similar replacement for polarizations, in the remainder of this paper. However, for the sake of clarity of presentation, this notation has been suppressed in the main body of the paper.

Accordingly, we provisionally write $E=E(u, \mathcal{E}, \eta)$ as a function of arguments $u_{m}, \mathcal{E}_{\alpha}$, and $\eta_{j}$, with the understanding that the notation of the Appendix supersedes the notation used here whenever strains and electric fields are simultaneously present. We then expand around a zero-field reference system as

$$
\begin{aligned}
E=E_{0} & +A_{m} u_{m}+A_{\alpha} \mathcal{E}_{\alpha}+A_{j} \eta_{j} \\
& +\frac{1}{2} B_{m n} u_{m} u_{n}+\frac{1}{2} B_{\alpha \beta} \mathcal{E}_{\alpha} \mathcal{E}_{\beta}+\frac{1}{2} B_{j k} \eta_{j} \eta_{k} \\
& +B_{m \alpha} u_{m} \mathcal{E}_{\alpha}+B_{m j} u_{m} \eta_{j}+B_{\alpha j} \mathcal{E}_{\alpha} \eta_{j} \\
& + \text { terms of third and higher order }
\end{aligned}
$$

We use an implied-sum notation throughout. In this expansion, the first-order coefficients $A_{m}, A_{\alpha}$, and $A_{\mathrm{j}}$ encode the forces $\left(F_{m}=-\Omega_{0} A_{m}\right)$, polarizations $\left(P_{\alpha}=\right.$ $\left.-A_{\alpha}\right)$, and stresses $\left(\sigma_{j}=A_{j}\right)$, respectively. (Henceforth we shall assume that the atomic coordinates and strains are fully relaxed in the reference system, so that $A_{m}=A_{j}=0$.) The diagonal-block second-order coefficients $B_{m n}, B_{\alpha \beta}$ and $B_{j k}$ and off-diagonal second-order coefficients $B_{m \alpha}, B_{m j}$, and $B_{\alpha j}$ correspond to the forceconstant, elastic-constant, and susceptibility tensors, and to the Born-charge, internal-displacement, and piezoelectric tensors, respectively.

Inserting appropriate signs and cell-volume factors, the elementary second-derivative response-function tensors are defined as follows. The force-constant matrix

$$
K_{m n}=\left.\Omega_{0} \frac{\partial^{2} E}{\partial u_{m} \partial u_{n}}\right|_{\mathcal{E}, \eta}
$$

the frozen-ion dielectric susceptibility

$$
\bar{\chi}_{\alpha \beta}=-\left.\frac{\partial^{2} E}{\partial \mathcal{E}_{\alpha} \partial \mathcal{E}_{\beta}}\right|_{u, \eta},
$$

and the frozen-ion elastic tensor

$$
\bar{C}_{j k}=\left.\frac{\partial^{2} E}{\partial \eta_{j} \partial \eta_{k}}\right|_{u, \mathcal{E}}
$$


are the elementary diagonal-block tensors, while the offdiagonal blocks are the Born dynamical effective charge tensor

$$
Z_{m a}=-\left.\Omega_{0} \frac{\partial^{2} E}{\partial u_{m} \partial \mathcal{E}_{\alpha}}\right|_{\eta}
$$

the force-response internal-strain tensor

$$
\Lambda_{m j}=-\left.\Omega_{0} \frac{\partial^{2} E}{\partial u_{m} \partial \eta_{j}}\right|_{\mathcal{E}},
$$

and the frozen-ion piezoelectric tensor

$$
\bar{e}_{\alpha j}=-\left.\frac{\partial^{2} E}{\partial \mathcal{E}_{\alpha} \partial \eta_{j}}\right|_{u} .
$$

The bar on quantities $\bar{\chi}_{\alpha \beta}, \bar{C}_{j k}$, and $\bar{e}_{\alpha j}$ indicates a frozen-ion quantity, i.e., the fact that atomic coordinates are not allowed to relax as the electric field or homogeneous strain is applied. Note that the clamped-ion elastic tensor $\bar{C}_{j k}$ and piezoelectric tensor $\bar{e}_{\alpha j}$ are generally not physically relevant quantities, except in cases of high symmetry where atomic displacements do not occur to first order in strain. The clamped-ion susceptibility tensor $\bar{\chi}_{\alpha \beta}$ is the purely electronic one that is measured in response to $\mathrm{AC}$ or optical fields at frequencies well above the phonon-frequency range (corresponding to $\epsilon^{\infty}$ in the polariton language).

The force-response internal-strain tensor $\Lambda_{m j}$ must be distinguished from the displacement-response internalstrain tensor $\Gamma_{n j}=\Lambda_{m j}\left(K^{-1}\right)_{m n}$ that describes the firstorder displacements resulting from a first-order strain; both occur in the literature, frequently without careful differentiation. The piezoelectric tensor $e_{\alpha j}$ (often denoted alternatively as $c_{\alpha j}$ ) describes the change of polarization arising from a strain, or a stress arising from a change of $\mathcal{E}$-field, while the $d, g$, and $h$ piezoelectric tensors are defined under different constraints and have slightly different physical meanings. ${ }^{16}$ Finally, we remind the reader that there is some subtlety in the definition of the piezoelectric tensors related to the specification of the energy functional when both fields and strains are present, leading to a distinction between "proper" and "improper" piezoelectric constants ${ }^{15}$ as will be discussed more fully in the Appendix. Throughout this paper, we adopt the convention that all piezoelectric tensors are "proper" ones unless otherwise noted.

We shall refer to the quantities defined in Eqs. (3-8) as the "elementary" or "bare" response tensors. These are the quantities that will be calculated once and for all using the DFPT capabilities of a code package such as ABINIT. All of the derived tensor properties described in the following subsections can then be calculated from these using simple matrix manipulations, as we shall see.

\section{B. Relaxed-ion tensors}

Generally, the physical static response properties of interest must take into account the relaxations of the ionic coordinates. This becomes especially important for non-centrosymmetric polar systems, such as ferroelectric ones, where these various effects become coupled. Instead of "clamped-ion" quantities $\bar{\chi}, \bar{C}$ and $\bar{e}$ defined at fixed $u$, we can define the corresponding "relaxed-ion" or "dressed" response tensors $C, \chi$, and $e$ as follows. To develop expressions for these, we let

$$
\widetilde{E}(\eta, \mathcal{E})=\min _{u} E(u, \mathcal{E}, \eta)
$$

Referring back to Eq. (2), setting $\partial E / \partial u_{n}=0$, $\partial E / \partial \mathcal{E}_{\alpha}=0$, and $\partial E / \partial \eta_{j}=0$, and assuming that the reference configuration is one in which the forces $A_{m}$ vanish, we find

$$
0=B_{n m} u_{m}+B_{n \alpha} \mathcal{E}_{\alpha}+B_{n j} \eta_{j}
$$

from which it follows that

$$
u_{m}=-\left(B^{-1}\right)_{m n}\left[B_{n j} \eta_{j}+B_{n \alpha} \mathcal{E}_{\alpha}\right] .
$$

Defining

$$
\begin{gathered}
\chi_{\alpha \beta}=-\left.\frac{\partial^{2} \widetilde{E}}{\partial \mathcal{E}_{\alpha} \partial \mathcal{E}_{\beta}}\right|_{\eta}, \\
C_{j k}=\left.\frac{\partial^{2} \widetilde{E}}{\partial \eta_{j} \partial \eta_{k}}\right|_{\mathcal{E}}, \\
e_{\alpha j}=-\frac{\partial^{2} \widetilde{E}}{\partial \mathcal{E}_{\alpha} \partial \eta_{j}},
\end{gathered}
$$

and using Eqs. (3-8), we find that the physical relaxedion dielectric, elastic, and piezoelectric tensors become

$$
\begin{aligned}
\chi_{\alpha \beta} & =\bar{\chi}_{\alpha \beta}+\Omega_{0}^{-1} Z_{m \alpha}\left(K^{-1}\right)_{m n} Z_{n \beta}, \\
C_{j k} & =\bar{C}_{j k}-\Omega_{0}^{-1} \Lambda_{m j}\left(K^{-1}\right)_{m n} \Lambda_{n k} \\
e_{\alpha j} & =\bar{e}_{j \alpha}+\Omega_{0}^{-1} Z_{m \alpha}\left(K^{-1}\right)_{m n} \Lambda_{n j}
\end{aligned}
$$

respectively.

Note that Eqs. (14-16) cannot be naively evaluated as written because the force-constant matrix $K$ is singular, due to the fact that $K$ has three vanishing eigenvalues associated with translational symmetry. Moreover, in soft-mode systems, other eigenvalues may be close to zero, and care should be taken to distinguish these from the translational ones. For these reasons, we have implemented a careful procedure for obtaining the "pseudoinverse" of $K$; throughout these notes, whenever we refer to $K^{-1}$, we really mean the pseudo-inverse.

We proceed as follows. (i) We identify the threedimensional space of acoustic modes (i.e., uniform translations), and construct a $(3 N) \times(3 N)$ orthogonal matrix 
$U$ whose first three columns correspond to these translational modes; the remaining columns of $U$ are formed by Graham-Schmidt orthogonalization of the basis. (ii) We construct $K^{\prime}=U K U^{T}$, whose upper $3 \times 3$ block represents the acoustic subspace and therefore ought to be zero. (iii) We let $K_{\text {red }}^{\prime}$ be the lower $(3 N-3) \times(3 N-3)$ block of $K^{\prime}$, corresponding to the reduction to the complementary subspace of optical modes. (iv) We invert $K_{\text {red }}^{\prime}$ by standard means (taking appropriate measures in case this matrix is nearly singular, as when soft modes have nearly vanishing frequencies). Let the result be denoted as $\left(K^{-1}\right)_{\text {red }}^{\prime}$ (v) We pad $\left(K^{-1}\right)_{\text {red }}^{\prime}$ with zeros in the first three rows and columns to form the $(3 N) \times(3 N)$ matrix $\left(K^{-1}\right)^{\prime}$. (vi) Finally, we define the pseudo-inverse of $K$ to be $K^{-1}=U^{T}\left(K^{-1}\right)^{\prime} U$.

Thus, by construction, the resulting pseudo-inverse $K^{-1}$ is zero in the subspace of translational modes, and is the inverse of the original matrix in the complementary subspace. As a result, any time $K^{-1}$ is multiplied by another tensor, a pre-projection onto the complementary subspace of dimension $3 N-3$ is effectively carried out. In other words, the acoustic sum rule is effectively enforced in any operation involving $K^{-1}$.

\section{Other derived tensor quantities}

In the previous subsection, we showed how to obtain the static dielectric susceptibility tensor $\chi_{\alpha \beta}$, the elastic tensor $C_{j k}$, and the piezoelectric tensor $e_{\alpha j}$. These quantities are defined under conditions of controlled strain and electric field. From these three ingredients, it is straightforward to form many other useful tensor quantities describing physical properties defined under other constraints or boundary conditions, as we shall see in this section.

\section{Dielectric tensors}

The susceptibility tensor $\chi_{\alpha \beta}$ is defined at fixed (vanishing) strain; the corresponding dielectric tensor is

$$
\epsilon_{\alpha \beta}^{(\eta)}=\epsilon_{0}\left(\delta_{\alpha \beta}+\chi_{\alpha \beta}\right)
$$

where $\epsilon_{0}$ is the susceptibility of free space (SI units are used throughout) and the superscript $(\eta)$ indicates that the derivative is taken at fixed strain. Often one is interested instead in the free-stress dielectric tensor

$$
\epsilon_{\alpha \beta}^{(\sigma)}=\epsilon_{0}\left(\delta_{\alpha \beta}+\chi_{\alpha \beta}^{(\sigma)}\right)
$$

which incorporates the free-stress susceptibility $\chi^{(\sigma)}$. An expression for the latter is easily derived from the elastic enthalpy

$$
\widetilde{H}(\sigma, \mathcal{E})=\min _{\{\eta\}}\left[\widetilde{E}(\eta, \mathcal{E})-\eta_{j} \sigma_{j}\right]
$$

Following a line of reasoning similar to that leading from Eq. (9) to Eqs. (14-16) and setting $\sigma_{j}=0$, one obtains

$$
\chi_{\alpha \beta}^{(\sigma)}=\chi_{\alpha \beta}+e_{\alpha j}\left(C^{-1}\right)_{j k} e_{\beta k} .
$$

Typically, an AC dielectric measurement will access the true static susceptibility $\chi^{(\sigma)}$ as long as the frequency is much less than that of sample resonances (elongational, bending, or torsional modes), and $\chi^{(\eta)}$ at frequencies much higher than sample resonances (but much less than phonon frequencies).

Before leaving this subsection, we note that it is convenient to define inverse dielectric tensors

$$
\begin{aligned}
& \beta^{(\eta)}=\left(\epsilon^{(\eta)}\right)^{-1}, \\
& \beta^{(\sigma)}=\left(\epsilon^{(\sigma)}\right)^{-1}
\end{aligned}
$$

for later use.

\section{Elastic and compliance tensors}

The elastic tensor $C_{j k}$ defined in Sec. II B is the one defined under conditions of fixed (vanishing) electric field: $C_{j k}=C_{j k}^{(\mathcal{E})}$. It may sometimes be of interest to treat instead the elastic tensor $C_{j k}^{(D)}$ defined under conditions of fixed electric displacement field D. For example, in the case of a thin film of dielectric material sandwiched between much thicker layers of other insulating host materials, the electrostatic boundary conditions fix the component of $D$, not $\mathcal{E}$, normal to the interfaces. One readily obtains

$$
C_{j k}^{(D)}=C_{j k}^{(\mathcal{E})}+e_{\alpha j} \beta_{\alpha \beta}^{(\eta)} e_{\beta k}
$$

It is also straightforward to obtain the corresponding elastic compliance tensors either under zero $\mathcal{E}$-field

$$
S^{(\mathcal{E})}=\left(C^{(\mathcal{E})}\right)^{-1}
$$

or under zero $D$-field

$$
S^{(D)}=\left(C^{(D)}\right)^{-1}
$$

boundary conditions.

\section{Piezoelectric tensors}

The formulation of an energy functional appropriate to the simultaneous treatment of strains and electric fields is rather subtle, as discussed in the Appendix. There, we show that the proper (relaxed-ion) piezoelectric tensor $e_{j k}$ introduced in Sec. II B may be written as

$$
e_{\alpha j}=\left.\frac{\partial P_{\alpha}}{\partial \eta_{j}}\right|_{\mathcal{E}}
$$


or equivalently, by a thermodynamic relation, ${ }^{17,16}$

$$
e_{\alpha j}=-\left.\frac{\partial \sigma_{j}}{\partial \mathcal{E}_{\alpha}}\right|_{\eta},
$$

where it is understood (see Appendix) that in these and subsequent equations, $\mathbf{P}$ and $\mathcal{E}$ are to be interpreted as the reduced polarization $\mathbf{P}^{\prime}$ and electric field $\mathcal{E}^{\prime}$ and of Eqs. (A4) and (A5), respectively. This is done so that Eqs. (26) and (27) will yield the proper, rather than the improper, piezoelectric tensor. ${ }^{15}$

In view of Eq. (27), $e_{\alpha j}$ is sometimes referred to as a "piezoelectric stress constant." In any case, it is the natural piezoelectric constant defined under conditions of controlled $\mathcal{E}$ and $\eta$. On the other hand, the "piezoelectric strain constant" $d_{\alpha j}$, defined under conditions of controlled $\mathcal{E}$ and $\sigma$, is equally or even more commonly discussed in the literature; it is defined via

$$
d_{\alpha j}=\left.\frac{\partial \eta_{j}}{\partial \mathcal{E}_{\alpha}}\right|_{\sigma}
$$

or

$$
d_{\alpha j}=\left.\frac{\partial P_{\alpha}}{\partial \sigma_{j}}\right|_{\mathcal{E}}
$$

and is given in terms of $e$ via

$$
d_{\alpha j}=S_{j k}^{(\mathcal{E})} e_{\alpha k}
$$

as can again be derived from thermodynamic relations. ${ }^{17,16}$ Two other, less commonly used, piezoelectric tensors are $g_{\alpha j}$ and $h_{\alpha j}$, defined under conditions of fixed $(D, \sigma)$ and $(D, \eta)$, respectively, and given by

$$
\begin{aligned}
& g_{\alpha j}=\beta_{\alpha \beta}^{(\sigma)} d_{\beta j}, \\
& h_{\alpha j}=\beta_{\alpha \beta}^{(\eta)} e_{\beta j},
\end{aligned}
$$

where the $\beta$ are the inverse dielectric tensors defined in Eqs. (21-22). These have the properties

$$
\begin{aligned}
\delta \eta_{j} & =g_{\alpha j} \delta D_{\alpha}, \\
\delta \mathcal{E}_{\alpha} & =-g_{\alpha j} \delta \sigma_{j} \\
\delta \sigma_{j} & =-h_{\alpha j} \delta D_{\alpha}, \\
\delta \mathcal{E}_{\alpha} & =-h_{\alpha j} \delta \eta_{j} .
\end{aligned}
$$

\section{Piezoelectric coupling coefficients}

The most common definition of the piezoelectric coupling factor $k_{\alpha j}$ is given by ${ }^{16,18}$

$$
k_{\alpha j}=\frac{\left|d_{\alpha j}\right|}{\sqrt{\epsilon_{\alpha \alpha}^{(\sigma)} S_{j j}^{(\mathcal{E})}}} .
$$

This applies to the case where the field is applied only along $\alpha$ and the only non-zero stress is the one with Voigt label $j$. For example, $k_{33}$ is a dimensionless measure of the coupling of electric and strain degrees of freedom along the $\hat{z}$ axis. Roughly speaking, a coupling factor close to unity implies an excellent impedance match for the material used as an electromechanical transducer between the specified electric and elastic channels (a coupling factor greater than unity is forbidden by stability considerations). ${ }^{16}$

Note that $k_{\alpha j}$ in Eq. (34) does not transform like a tensor, and the usual implied sum notation does not apply to this equation. Instead, we can define a tensorially correct, dimensionless coupling tensor via

$$
\mathcal{K}=\left[\beta^{(\sigma)}\right]^{1 / 2} \cdot d \cdot\left[C^{(\mathcal{E})}\right]^{1 / 2},
$$

where an obvious matrix-product notation is used. The standard "singular-value decomposition" can be used to write $\mathcal{K}$ as

$$
\mathcal{K}=\mathcal{U} \cdot\left(\begin{array}{cccccc}
\tilde{k}_{1} & 0 & 0 & 0 & 0 & 0 \\
0 & \tilde{k}_{2} & 0 & 0 & 0 & 0 \\
0 & 0 & \tilde{k}_{3} & 0 & 0 & 0
\end{array}\right) \cdot \mathcal{V}^{T}
$$

defined by requiring that $\mathcal{U}$ and $\mathcal{V}$ be orthogonal $3 \times 3$ and $6 \times 6$ matrices respectively, and that the $\widetilde{k}_{\nu}$ should be positive. (Alternatively, the $\tilde{k}_{\nu}^{2}$ can be determined as the eigenvalues of the $3 \times 3$ symmetric matrix $\mathcal{K} \mathcal{K}^{T}=$ $\left[\beta^{(\sigma)}\right]^{1 / 2} d C^{(\mathcal{E})} d^{T}\left[\beta^{(\sigma)}\right]^{1 / 2}$.) For each singular value, the corresponding columns of $\mathcal{U}$ and $\mathcal{V}$ give the pattern of electric field and of strain, respectively, that are directly coupled to one another by $\mathcal{K}$.

The coupling factors can be related to differences between dielectric or compliance tensors defined under different boundary conditions. Starting from Eqs. (18-20) and Eqs. (23-25), one can show

$$
\begin{aligned}
\epsilon^{(\sigma)}-\epsilon^{(\eta)} & =d C^{(\mathcal{E})} d^{T}=\left[\epsilon^{(\sigma)}\right]^{1 / 2} \mathcal{K} \mathcal{K}^{T}\left[\epsilon^{(\sigma)}\right]^{1 / 2} \\
S^{(\mathcal{E})}-S^{(D)} & =d^{T} \beta^{(\sigma)} d=\left[S^{(\mathcal{E})}\right]^{1 / 2} \mathcal{K}^{T} \mathcal{K}\left[S^{(\mathcal{E})}\right]^{1 / 2}
\end{aligned}
$$

Specializing to high-symmetry situations in which $\epsilon$ is necessarily diagonal, one finds, for example,

$$
\frac{\epsilon_{\alpha \alpha}^{(\sigma)}-\epsilon_{\alpha \alpha}^{(\eta)}}{\epsilon_{\alpha \alpha}^{(\sigma)}}=\tilde{k}_{\alpha}^{2} .
$$

\section{METHODS AND DETAILS OF THE CALCULATIONS}

Our ab-initio calculations were carried out using the ABINIT code package. ${ }^{7,19}$ Specifically, we first carried out full structural relaxations for both materials. Next, response-function calculations were carried out in order to obtain first derivatives of the occupied wavefunctions with respect to the perturbations of atomic displacements (i.e., phonons at $q=0$ ), uniform electric field, and strain. These were then used to compute the elementary second-derivative response-function tensors, Eqs. (3-8), 
of Sec. II A. Except for the diagonal elements of some elementary tensors, this was done using a non-variational expression that only requires input of one of the two corresponding wavefunction derivatives. ${ }^{2}$ (As for the "mixed derivative" tensors $\Lambda, e$ and $Z$, strain derivatives were used for $\Lambda$ and $e$, and displacement derivatives were used for $Z .^{20}$ ) Finally, from these elementary response tensors, the various secondary response tensors of Secs. II B and II C are obtained according to the formulas given there. All calculations are at zero temperature.

The DFT and DFPT calculations for $\mathrm{ZnO}$ and $\mathrm{BaTiO}_{3}$ were carried out using Troullier-Martins pseudopotentials $^{21}$ and a plane-wave energy cutoff of 50 hartree. The $\mathrm{Zn}$ pseudopotential includes the $3 d$ electrons in the valence, as this has been shown to be important for accurate results. ${ }^{22}$ An $8 \times 8 \times 8$ and $6 \times 6 \times 6$ Brillouin-zone k-point sampling were used for $\mathrm{ZnO}$ and $\mathrm{BaTiO}_{3}$ respectively. The exchange and correlation effects were treated within the local-density approximation (LDA) in the Ceperley-Alder ${ }^{23}$ form with the PerdewWang $^{24}$ parameterization.

Finally, we made one additional modification in the case of $\mathrm{BaTiO}_{3}$, where it is well known that the usual underestimation of the equilibrium lattice constant associated with the local-density approximation has an unusually profound influence on the ferroelectric distortion, which is very sensitive to cell volume. ${ }^{25}$ Therefore, to get more physically meaningful results, we carried out the initial structural relaxation with the cell volume constrained to be that of the experimental structure at zero temperature. This is similar in spirit to the use of a "negative fictitious pressure" that is a standard feature of many first-principles based studies of ferroelectric perovskite materials. $^{25}$

\section{RESULTS FOR TWO SAMPLE SYSTEMS: $\mathrm{ZnO}$ AND $\mathrm{BaTiO}_{3}$}

In this section, we consider two paradigmatic systems, wurtzite $\mathrm{ZnO}$ and rhombohedral $\mathrm{BaTiO}_{3}$. The electromechanical properties of $\mathrm{ZnO}$ make it a widely used material in mechanical actuators and piezoelectric sensor applications, while $\mathrm{BaTiO}_{3}$ is a prototypical perovskite ferroelectric material. It is of particular interest to compare and contrast the behavior of these two materials in view of the fact that $\mathrm{BaTiO}_{3}$ is a soft-mode system, while $\mathrm{ZnO}$ is not. This may help provide insight into the role of the soft mode, which can be expected to lead to enhanced piezoelectric and dielectric response and enhanced couplings. We first describe the results of our ground-state DFT calculations, and then present the results for the various linear-response tensors as defined in Sec. II.

Because $\mathrm{ZnO}$ is not a soft-mode system, its properties depend only weakly on temperature, so that it is not unreasonable to compare room-temperature experimental results with zero-temperature theory. $\mathrm{BaTiO}_{3}$ is a differ-
TABLE I. Structural parameters of ZnO. Lattice constants $a$ and $c$ in $\AA ; u$ is dimensionless internal parameter.

\begin{tabular}{lcclc}
\hline \hline & $a$ & $c$ & $c / a$ & $u$ \\
\hline Present work $^{\mathrm{a}}$ & 3.197 & 5.166 & 1.616 & 0.380 \\
Previous theory $^{\mathrm{a}}$ & 3.286 & 5.241 & 1.595 & 0.383 \\
Expt. $^{\mathrm{b}}$ & 3.250 & 5.210 & 1.602 & 0.382 \\
\hline \hline
\end{tabular}

${ }^{\mathrm{a}}$ Ref. 28.

${ }^{\mathrm{b}}$ Ref. 29 .

ent matter, as its properties depend crucially on temperature. The room-temperature tetragonal phase has indeed been thoroughly studied, and as a result, its dielectric, elastic, and piezoelectric constants have been systematically measured. ${ }^{26,27}$ However, there are formidable difficulties associated with preparing single-crystal, singledomain samples of the low-temperature rhombohedral phase, and of carrying out dielectric and elastic measurements on such samples at low temperature under welldefined electric and elastic boundary conditions. As a result, almost no reliable experimental values are available for the corresponding materials constants at very low temperature. Therefore, for the purposes of this paper, we have adopted the approach of providing comparisons with experiment for $\mathrm{ZnO}$ wherever possible to benchmark our approach, and of presenting our calculations of the low-temperature properties of $\mathrm{BaTiO}_{3}$ as predictions for a system that is difficult to characterize experimentally.

\section{A. Relaxed structural properties}

$$
\text { 1. } \mathrm{ZnO}
$$

The ground state of $\mathrm{ZnO}$ is a tetrahedrally coordinated wurtzite structure (space group $P 6_{3} m c$, point group $C_{6 v}$ ) with four atoms per unit cell. The structure is determined by three parameters: the hexagonal edge $a$, the height of the prism $c$, and the internal parameter $u$. The structural results from our full relaxation are given in Table I. For comparison, an ideal wurtzite with exactly tetrahedral angles and equal-length bonds would have $u=3 / 8$ and $c / a=\sqrt{8 / 3}$. As is typical of DFT calculations, we find that the lattice constants are underestimated by $1-2 \%$.

TABLE II. Relaxed structure of rhombohedral $\mathrm{BaTiO}_{3}$. Lattice constant $a$ and atomic displacements $\Delta$ (relative to ideal cubic positions) in $\AA$; rhombohedral angle $\theta$ in degrees.

\begin{tabular}{lrrrrr}
\hline \hline & $a$ & $\theta$ & $\Delta_{z}(\mathrm{Ti})$ & $\Delta_{x}(\mathrm{O})$ & $\Delta_{z}(\mathrm{O})$ \\
\hline Theory & 4.00 & 89.85 & 0.043 & -0.049 & -0.077 \\
Expt. $^{\text {a }}$ & 4.00 & 89.90 & $0.052 \pm 12$ & $-0.044 \pm 8$ & $-0.072 \pm 8$ \\
\hline \hline
\end{tabular}

${ }^{\mathrm{a}}$ Ref. 30. 
TABLE III. Frequencies(in $\mathrm{cm}^{-1}$ ) of the zone-center optical phonon modes in wurtzite $\mathrm{ZnO}$.

\begin{tabular}{lccc}
\hline \hline symmetry character & Theory & Expt. $^{31}$ & Expt $^{32}$ \\
\hline $\mathrm{A}_{1}(\mathrm{TO})$ & 390 & 380 & 380 \\
$\mathrm{~A}_{1}(\mathrm{LO})$ & 548 & 574 & 579 \\
$\mathrm{~B}_{1}$ & 261 & - & - \\
$\mathrm{E}_{1}(\mathrm{TO})$ & 409 & 407 & 413 \\
$\mathrm{E}_{1}(\mathrm{LO})$ & 552 & 583 & 591 \\
$\mathrm{E}_{2}$ & 91 & 101 & 101 \\
$\mathrm{E}_{2}$ & 440 & 437 & 444 \\
\hline \hline
\end{tabular}

\section{2. $\mathrm{BaTiO}_{3}$}

$\mathrm{BaTiO}_{3}$ is a prototypical example of the class of perovskite ferroelectric materials. These materials normally have the paraelectric cubic perovskite structure at high temperature, but then undergo a ferroelectric instability as the temperature is reduced. $\mathrm{BaTiO}_{3}$ actually goes through a series of three ferroelectric phase transitions as the symmetry is first tetragonal, then orthorhombic, and then rhombohedral, with polarization respectively along [100], [110], and [111], with decreasing temperature. The ground-state rhombohedral structure (space group $R 3 \mathrm{~m}$, point group $C_{3 v}$ ) is fully determined by its lattice constant, rhombohedral angle, and the symmetry-allowed internal atomic displacements along the [111] direction. We represent the rhombohedral phase in the hexagonal coordinate system, in which the $z$ axis is along the previous [111] direction. Table II lists the structural parameters of our relaxed $\mathrm{BaTiO}_{3}$, in which we constrained the atomic volume to be equal to the experimental one as explained in Sec. III. The remaining structural parameters can be seen to be in good agreement with experiment.

\section{B. Displacement response tensors}

$$
\text { 1. } \mathrm{ZnO}
$$

Wurtzite $\mathrm{ZnO}$ belongs to space group $P 6_{3} m c\left(C_{6 v}^{4}\right)$. Standard group-theory analysis shows that the $\Gamma$-point phonon modes can be decomposed as

$$
\Gamma_{\text {opt }}=A_{1} \oplus 2 B_{1} \oplus E_{1} \oplus 2 E_{2}
$$

in which the $A_{1}$ and $E_{1}$ modes are both Raman and IR active, while the nonpolar $E_{2}$ modes are Raman active only and $B_{1}$ modes are silent. Shown in Table III are our computed phonon frequencies compared with two experimental results, showing good agreement with experiment.

Because of the wurtzite symmetry of $\mathrm{ZnO}$, the effective charge tensor $Z$ has only two independent elements, while the force-response internal-strain tensor $\Lambda$ has only
TABLE IV. Independent elements of the Born effective charge tensor $Z$ (in units of $e$ ) and of the force-response internal strain tensor (hartree/bohr) for wurtzite $\mathrm{ZnO}$.

\begin{tabular}{lccc}
\hline \hline$Z_{x z}(\mathrm{Zn})$ & $Z_{z z}(\mathrm{Zn})$ & & \\
2.135 & 2.163 & & \\
\hline$\Lambda_{x 5}(\mathrm{Zn})$ & $\Lambda_{x 6}(\mathrm{Zn})$ & $\Lambda_{z 3}(\mathrm{Zn})$ & $\Lambda_{y 1}(\mathrm{O})$ \\
-9.5 & -15.0 & 18.7 & -16.7 \\
\hline \hline
\end{tabular}

four independent elements. We present results for both tensors in Table IV. For this semiconductor material, it can be seen that the effective charge is very close to the nominal ionic charge.

\section{2. $\mathrm{BaTiO}_{3}$}

The low-temperature phase of $\mathrm{BaTiO}_{3}$ has a rhombohedral structure which belongs to the $R 3 m$ space group. According to a group-theory analysis, the zone-center phonon frequencies can be decomposed as

$$
\Gamma_{\mathrm{opt}}=3 \mathrm{~A}_{1} \oplus 4 \mathrm{E} \oplus \mathrm{A}_{2} .
$$

The $\mathrm{A}_{1}$ and $\mathrm{E}$ modes are both $\mathrm{IR}$ and Raman active, while the $\mathrm{A}_{2}$ mode is silent. Table $\mathrm{V}$ gives the calculated phonon frequencies at the $\Gamma$ point. (The $\mathrm{A}_{2}$ mode at $278 \mathrm{~cm}^{-1}$ and the $\mathrm{E}$ modes at $293 \mathrm{~cm}^{-1}$ are the ones derived from the silent $\mathrm{F}_{2 u}$ modes of the undistorted $\mathrm{cu}-$ bic structure; because the distortion is weak, the LO-TO splitting of these $\mathrm{E}$ modes is negligible.) The results are very similar to those of the previous theoretical study of Ghosez. ${ }^{33}$ While we are not aware of detailed experimental information about phonon frequencies at very low temperature, we note that measurements just below the orthorhombic to rhombohedral phase transition temperature indicate phonon frequencies in three regions (100$300 \mathrm{~cm}^{-1}, 480-580 \mathrm{~cm}^{-1}$, and $680-750 \mathrm{~cm}^{-1}$ ) in qualitative agreement with our zero-temperature calculations.

We also calculated the atomic Born effective charges for this phase, but in view of the lower symmetry and larger number of independent elements, we have not listed them all here (our results are again very similar

TABLE V. Phonon frequencies $\left(\right.$ in $\mathrm{cm}^{-1}$ ) at the $\Gamma$ point for rhombohedral $\mathrm{BaTiO}_{3}$.

\begin{tabular}{lclc}
\hline \hline Phonon mode & Frequency & Phonon mode & Frequency \\
\hline $\mathrm{A}_{1}(\mathrm{TO} 1)$ & 169 & $\mathrm{E}(\mathrm{TO} 1)$ & 164 \\
$\mathrm{~A}_{1}(\mathrm{LO} 1)$ & 179 & $\mathrm{E}(\mathrm{LO} 1)$ & 175 \\
$\mathrm{~A}_{1}(\mathrm{TO} 2)$ & 255 & $\mathrm{E}(\mathrm{TO} 2)$ & 206 \\
$\mathrm{~A}_{1}(\mathrm{LO} 2)$ & 460 & $\mathrm{E}(\mathrm{LO} 2)$ & 443 \\
$\mathrm{~A}_{1}$ (TO3) & 511 & $\mathrm{E}(\mathrm{TO} 3)$ & 472 \\
$\mathrm{~A}_{1}(\mathrm{LO} 3)$ & 677 & $\mathrm{E}(\mathrm{LO} 3)$ & 687 \\
$\mathrm{~A}_{2}$ & 278 & $\mathrm{E}$ & 293 \\
\hline \hline
\end{tabular}


TABLE VI. Dielectric tensors of $\mathrm{ZnO}$ and $\mathrm{BaTiO}_{3}$ (in units of $\left.\epsilon_{0}\right)$.

\begin{tabular}{lcrrrrr}
\hline \hline & \multicolumn{4}{c}{ Present theory } & \multicolumn{2}{c}{ Experiment } \\
& Index & $\bar{\epsilon}$ & \multicolumn{1}{c}{$\epsilon$} & \multicolumn{1}{c}{$\epsilon^{(\sigma)}$} & \multicolumn{1}{c}{} & $\epsilon$ \\
\hline $\mathrm{ZnO}$ & 11 & 5.76 & 10.31 & 11.09 & $3.70^{\mathrm{a}}$ & $7.77^{\mathrm{a}}$ \\
& 33 & 5.12 & 10.27 & 12.67 & $3.78^{\mathrm{a}}$ & $8.91^{\mathrm{a}}$ \\
$\mathrm{BaTiO}_{3}$ & 11 & 6.20 & 68.75 & 264.75 & $6.19^{\mathrm{b}}$ & - \\
& 33 & 5.79 & 37.44 & 49.51 & $5.88^{\mathrm{b}}$ & - \\
\hline \hline
\end{tabular}

${ }^{\mathrm{a}}$ Ref. 34 .

${ }^{\mathrm{b}}$ Ref. 35 .

to those of Ref. 33). The cation results are easily given as $Z_{x x}(\mathrm{Ba})=Z_{y y}(\mathrm{Ba})=2.78, Z_{z z}(\mathrm{Ba})=2.74, Z_{x x}(\mathrm{Ti})$ $=Z_{y y}(\mathrm{Ti})=6.64$, and $Z_{z z}(\mathrm{Ti})=5.83$. The effective charge tensors are non-diagonal and non-symmetric for the oxygens; we mention only that the eigenvalues of the symmetric parts of these tensors cluster around -2 and -5 , i.e., not much changed from their cubic-phase values. Similarly, we have computed the full internal-strain tensor $\Lambda$ for rhombohedral $\mathrm{BaTiO}_{3}$, but we have chosen not to present the details here because of the complicated form of this tensor involving a large number of independent elements.

\section{Dielectric tensors}

We now turn to a discussion of the computed dielectric tensors for wurtzite $\mathrm{ZnO}$ and rhombohedral $\mathrm{BaTiO}_{3}$, which are presented in Table VI. Because of the high point-group symmetry, the dielectric tensors have only two independent elements. Recall that the clampedion tensor $\bar{\epsilon}$, the fixed-strain relaxed-ion tensor $\epsilon$, and the free-stress relaxed-ion tensor $\epsilon^{(\sigma)}$ are defined through Eqs. (14) and (17-20). While the results for the purely electronic dielectric tensors $\bar{\epsilon}$ are in good agreement with experiment for $\mathrm{BaTiO}_{3}$, we find that our LDA theory significantly overestimates the electronic dielectric response of $\mathrm{ZnO}$. Hill and Waghmare, ${ }^{22}$ also using an LDA pseudopotential approach, found $\bar{\epsilon}_{33}=4.39$, not as large as our 5.76, but still much larger than the experimental 3.70. At least some of this overestimate is undoubtedly attributable to the LDA (and is connected with the underestimate of the gap in LDA), but the choice of pseudopotentials also seems to play a role. The computed lattice contributions $\epsilon_{11}-\bar{\epsilon}_{11}=4.55$ and $\epsilon_{33}-\bar{\epsilon}_{33}=5.15$ are in better agreement with the experimental values of 4.07 and 5.13 respectively.

While the clamped-ion tensors $\bar{\epsilon}$ are not so different for these two materials, the lattice contribution is clearly much bigger for the $\mathrm{BaTiO}_{3}$ case. That is, while the lattice contribution $(\epsilon-\bar{\epsilon})$ is about the same size as the purely electronic one $(\bar{\epsilon})$ for $\mathrm{ZnO}$, it is almost 10 times larger in $\mathrm{BaTiO}_{3}$. This difference clearly reflects the fact there is a soft ferroelectric mode present in the latter material. (Here, we use "soft" in the sense of a mode that has a small, but positive frequency; it is, of course, closely related to the imaginary-frequency unstable mode computed for the cubic structure, which condenses out to form the ferroelectric rhombohedral phase.) In the semiconductor $\mathrm{ZnO}$, on the other hand, no such soft mode is present.

The last column presents our results for the free-stress dielectric tensors $\epsilon^{(\sigma)}$ that are related to the fixed-strain tensors $\epsilon$ via Eq. (20). The tensors $\epsilon$ and $\epsilon^{(\sigma)}$ are the same in higher-symmetry crystals, but in the presence of piezoelectric coupling, they are, in general, different. The free-stress tensors are always larger than the fixed-strain ones because the additional strain relaxation occurs so as to allow further polarization to develop in the direction of the applied field. We can see that the changes are modest for $\mathrm{ZnO}$ (on the order of 10-20\%), which is not a softmode system. On the other hand, they are much more profound for the case of $\mathrm{BaTiO}_{3}$, where most notably an order-of-magnitude change occurs for $\epsilon_{11}$. This is related to the large value of the piezoelectric coupling factor $k_{15}$, as we will see later in Sec. IIC4. Essentially, it arises because the polarization vector is rather soft with respect to rotation away from the $z$ axis, so that the electric susceptibility is large in the $x-y$ plane.

\section{Elastic tensors}

We now consider the various elastic tensors. Recall that the clamped-ion elastic tensor $\bar{C}$ of Eq. (5) is just the second derivative of the unit-cell energy with respect to homogeneous strains, without allowing for internal structural relaxations, while the physical elastic tensor $C$ of Eq. (15) does include such relaxations. This tensor $C$ (written more explicitly as $C^{(\mathcal{E})}$ ) usually defined under conditions of fixed macroscopic electric field, but it is sometimes of interest to consider the elastic tensor $C^{(D)}$ of Eq. (23) defined instead under conditions of fixed electric displacement field. These are identical for highersymmetry (e.g., centrosymmetric) crystals, but that is not the case here. The compliance tensors $S$ are defined as the inverses of the corresponding elastic tensors $C$.

The results of our calculations of these tensors are displayed in Table VII and VIII for $\mathrm{ZnO}$ and $\mathrm{BaTiO}_{3}$ respectively. The lower point-group symmetry of $\mathrm{BaTiO}_{3}$ is reflected in the presence of an additional symmetry-allowed element $C_{14}$. Actually, there are only five independent elements for $\mathrm{ZnO}$, since $C_{66}=\left(C_{11}-C_{12}\right) / 2$ and $S_{66}=$ $2\left(S_{11}-S_{12}\right)$ by symmetry. ${ }^{17}$ Similarly, there are really only six independent elements for $\mathrm{BaTiO}_{3}$; in addition to the same relation, one also has $C_{66}=\left(C_{11}-C_{12}\right) / 2$, $S_{66}=2\left(S_{11}-S_{12}\right), C_{56}=C_{14}$, and $S_{56}=2 C_{14}$. Our results for the elastic constants of $\mathrm{ZnO}$ can be seen to be in good agreement with previous theory and with experiment (last columns of Table VII). 
TABLE VII. Clamped-ion $(\bar{C})$ and relaxed-ion $(C)$ elastic tensors at constant $\mathcal{E}$, relaxed ion $\left(C^{(D)}\right)$ elastic tensor at constant $D(\mathrm{GPa})$, and corresponding compliance tensors $\left(\bar{S}, S\right.$, and $\left.S^{(D)}\right)\left(\mathrm{TPa}^{-1}\right)$, for wurtzite ZnO. Previous theoretical and experimental results are also given for $C$ for comparison.

\begin{tabular}{|c|c|c|c|c|c|c|c|c|}
\hline \multirow[b]{2}{*}{ Index } & \multicolumn{6}{|c|}{ Present theory } & \multirow{2}{*}{$\begin{array}{c}\text { Theo. }^{\mathrm{a}} \\
C\end{array}$} & \multirow{2}{*}{$\begin{array}{c}\text { Expt. }^{\mathrm{b}} \\
C\end{array}$} \\
\hline & $\bar{C}$ & $C$ & $C^{(D)}$ & $\bar{S}$ & $S$ & $S^{(D)}$ & & \\
\hline$\overline{11}$ & 305 & 226 & 231 & 3.86 & 7.79 & 7.56 & 246 & 209 \\
\hline 12 & 107 & 139 & 144 & -1.20 & -3.63 & -3.93 & 127 & 120 \\
\hline 13 & 77 & 123 & 114 & -0.61 & -2.12 & -1.58 & 105 & 104 \\
\hline 33 & 333 & 242 & 260 & 3.29 & 6.28 & 5.23 & 246 & 211 \\
\hline 44 & 62 & 40 & 43 & 16.23 & 24.69 & 23.21 & 56 & 44 \\
\hline 66 & 99 & 44 & 44 & 10.12 & 22.84 & 22.73 & - & 一 \\
\hline
\end{tabular}

${ }^{\mathrm{a}}$ Ref. 28.

${ }^{\mathrm{b}}$ Ref. 36 .

TABLE VIII. Clamped-ion $(\bar{C})$ and relaxed-ion $(C)$ elastic tensors at constant $\mathcal{E}$, relaxed ion $\left(C^{(D)}\right)$ elastic tensor at constant $D(\mathrm{GPa})$, and corresponding compliance tensors $(\bar{S}$, $S$, and $\left.S^{(D)}\right)\left(\mathrm{TPa}^{-1}\right)$, for rhombohedral $\mathrm{BaTiO}_{3}$.

\begin{tabular}{lcccrrr}
\hline \hline Index & $\bar{C}$ & $C$ & $C^{(D)}$ & \multicolumn{1}{c}{$\bar{S}$} & \multicolumn{1}{c}{$S$} & \multicolumn{1}{c}{$S^{(D)}$} \\
\hline 11 & 349 & 277 & 318 & 3.32 & 5.85 & 3.65 \\
12 & 106 & 79 & 93 & -0.82 & -2.94 & -0.95 \\
13 & 96 & 41 & 81 & -0.72 & -0.45 & -0.68 \\
14 & 8.4 & 45 & 19 & -0.31 & -8.17 & -0.89 \\
33 & 334 & 264 & 323 & 3.41 & 3.93 & 3.44 \\
44 & 110 & 48 & 97 & 9.12 & 35.85 & 10.63 \\
65 & 8.3 & 45 & 19 & -0.63 & -16.33 & -1.78 \\
66 & 121 & 99 & 113 & 8.28 & 17.58 & 9.18 \\
\hline \hline
\end{tabular}

We notice that the physical elastic $C_{j k}$ are generally smaller than the frozen-ion ones $\bar{C}_{j k}$ (at least for diagonal elements), since the additional internal relaxation allows some of the stress to be relieved. By the same token, diagonal $S$ values are larger than $\bar{S}$ ones, reflecting the increased compliance allowed by the relaxation of the atomic coordinates. As for the dielectric constants, the differences are substantially smaller for $\mathrm{ZnO}$ than for $\mathrm{BaTiO}_{3}$, as a result of the soft-mode contribution in the latter material. The constraint of fixed electric displacement field has the effect of suppressing some of this internal relaxation (for essentially the same reason that longitudinal optical phonons are stiffer than transverse optical ones). This additional stiffness results in larger diagonal $C^{(D)}$ values than $C$ values, and lower diagonal $S^{(D)}$ values than $S$ values. However, the differences between $C^{(D)}$ and $C$ tensors are generally smaller than the differences between $C$ and $\bar{C}$ tensors, especially for $\mathrm{ZnO}$.

\section{E. Piezoelectric tensors}

The bare (or "frozen-ion") piezoelectric tensor $\bar{e}_{\alpha j}$ is just given by the mixed second derivatives of unit cell energy with respect to electric field $\mathcal{E}_{\alpha}$ and strain $\eta_{j}$, deforming internal atomic coordinates in strict proportion to the homogeneous strain. The full piezoelectric tensor $e_{\alpha j}$ also takes into account the contributions from the lattice, as described in Eq. 16. The total number of independent piezoelectric tensor members is determined by the point group of material. Rhombohedral $\mathrm{BaTiO}_{3}$ (point group $\mathrm{C}_{3 v}$ ) has a lower symmetry than that of wurtzite $\mathrm{ZnO}\left(\mathrm{C}_{6 v}\right)$, so we may expect more independent elements in the former. Indeed, a symmetry analysis ${ }^{17}$ shows that $\mathrm{ZnO}$ has only three independent tensor elements, namely $e_{31}$ and $e_{33}$ describing polarization along the $c$ axis induced by uniaxial $c$-axis or biaxial $a b$-plane strains, while $e_{24}$ describes the polarization induced by shear strains. For $\mathrm{BaTiO}_{3}$, the symmetry is slightly lower, and as a result there is a fourth independent tensor element in this case.

In Table IX and X, we present our results for piezoelectric tensors for these two materials. We also also give the results for the $d_{\alpha j}$ tensor as defined in Eq. 30. Our results for the $e_{\alpha j}$ matrix for $\mathrm{ZnO}$ are consistent with the previous theory. ${ }^{22,37,38}$ (Table X shows five tensor elements for $\mathrm{BaTiO}_{3}$, not four, but in fact $e_{21}=e_{16}$ and $2 d_{21}=d_{16}$ by symmetry. ${ }^{17}$ )

Recall that the frozen-ion and relaxed-ion piezoelectric tensors are defined by Eqs. (8) and (16), in which the relaxed-ion piezoelectric tensor incorporates contributions from lattice relaxation. For the same reason as discussed previously for the case of the dielectric and elastic tensors, the difference between the above two tensors (e.g. $\bar{e}$ and $e$ ) is much bigger for $\mathrm{BaTiO}_{3}$ than for $\mathrm{ZnO}$, as expected from the presence of the soft mode in the perovskite material. Also, note that the electronic and lattice contributions have opposite signs, with the lattice contribution being the larger of the two, as is common for other dielectric materials. 
TABLE IX. Clamped-ion $\bar{e}\left(\mathrm{C} / \mathrm{m}^{2}\right)$, relaxed-ion $e\left(\mathrm{C} / \mathrm{m}^{2}\right)$, and relaxed-ion $d(\mathrm{pC} / \mathrm{N})$ piezoelectric tensors for wurtzite ZnO. Previous theoretical and experimental results are also given for $e$ and $d$ for comparison.

\begin{tabular}{lcccccc}
\hline \hline & & Present theory & \multicolumn{3}{c}{ Theo. $^{\text {a }}$} & \multicolumn{2}{c}{ Expt. $^{\text {b }}$} & $d$ \\
Index & $\bar{e}$ & $e$ & $d$ & $e$ & -3.7 & -0.62 \\
31 & 0.37 & -0.67 & -5.5 & -0.55 & -5.1 \\
33 & -0.75 & 1.28 & 10.9 & 1.19 & 8.0 & 0.96 \\
15 & 0.39 & -0.53 & -13.1 & -0.46 & -8.2 & -0.37 \\
\hline \hline
\end{tabular}

${ }^{\mathrm{a}}$ Ref. 28 .

${ }^{\mathrm{b}}$ Ref. 36 .

TABLE X. Clamped-ion $\bar{e}\left(\mathrm{C} / \mathrm{m}^{2}\right)$, relaxed-ion $e\left(\mathrm{C} / \mathrm{m}^{2}\right)$, and relaxed-ion $d(\mathrm{pC} / \mathrm{N})$ piezoelectric tensors for rhombohedral $\mathrm{BaTiO}_{3}$.

\begin{tabular}{lrrr}
\hline \hline Index & $\bar{e}$ & $e$ & $d$ \\
\hline 21 & -0.23 & 2.91 & 70.1 \\
31 & 0.05 & -3.03 & -6.8 \\
33 & -0.19 & -4.44 & -14.7 \\
15 & 0.18 & -5.45 & -243.2 \\
16 & -0.23 & 2.91 & 140.2 \\
\hline \hline
\end{tabular}

In view of this partial cancellation of terms of opposite sign, accurate calculations of $e$ and $d$ coefficients are particularly delicate. We find that our results for the 31 and 33 elements of the $e$ and $d$ coefficients of $\mathrm{ZnO}$ are in reasonably good agreement with experiment (slightly better than previous Hartree-Fock calculations ${ }^{28}$ ), whereas we somewhat overestimate the shear coefficients $e_{15}$ and $d_{15}$ (slightly more so than in the Hartree-Fock theory ${ }^{28}$ ).

\section{F. Electromechanical coupling constants}

We compute and present in Table XI the piezoelectric coupling factors $k_{33}, k_{31}$, and $k_{15}$ defined in Eq. (34) for both $\mathrm{ZnO}$ and $\mathrm{BaTiO}_{3}$. We also calculate the singular values $\tilde{k}_{\nu}$ of the $\mathcal{K}$ matrix of Eq. (35). Because of the axial symmetry, these are arranged into a pair of degenerate values $\tilde{\kappa}_{1}=\tilde{k}_{2}$ corresponding to in-plane electric fields, and a $\tilde{k}_{3}$ corresponding to axial fields. (In fact, due to the symmetry, $\tilde{k}_{\nu}$ cab be calculated in practice as just $\left[\beta_{\nu \nu}^{(\sigma)}\left(d \cdot C^{(\mathcal{E})} \cdot d^{T}\right)_{\nu \nu}\right]^{1 / 2}$.) These $\tilde{k}_{\nu}$ values are also given in Table XI.

Roughly speaking, the couplings given in the first three lines of Table XI are associated with symmetrypreserving "polarization stretching" degrees of freedom, while those in the last two lines correspond to "polarization rotation" modes. Note that $k_{15}=\tilde{k}_{1}$ for $\mathrm{ZnO}$ but not for $\mathrm{BaTiO}_{3}$; this is a feature of symmetry, arising because an electric field $\mathcal{E}_{1}$ couples uniquely to $\eta_{5}$ in $\mathrm{ZnO}$, but also to $\eta_{6}$ in $\mathrm{BaTiO}_{3}$. Also, we can see that $\tilde{k}_{1} \geq k_{15}$ and $\tilde{k}_{4} \geq \max \left(k_{33}, k_{31}\right)$ in both materials, since $\tilde{\tilde{k}}$ describes the optimal coupling between electric and elastic
TABLE XI. Dimensionless piezoelectric coupling factors. The first three correspond to $\mathcal{E}$-fields longitudinal to the crystal axis; the last two are transverse. Coupling constants $\tilde{k}_{1}$ and $\tilde{k}_{3}$ are obtained from singular-value analysis of the coupling tensor $\mathcal{K}$ (see text).

\begin{tabular}{lcc}
\hline \hline & $\mathrm{ZnO}$ & $\mathrm{BaTiO}_{3}$ \\
\hline$k_{33}$ & 0.41 & 0.35 \\
$k_{31}$ & 0.19 & 0.13 \\
$\tilde{k}_{3}$ & 0.44 & 0.49 \\
$k_{15}$ & 0.27 & 0.84 \\
$\tilde{k}_{1}$ & 0.27 & 0.86 \\
\hline \hline
\end{tabular}

channels.

Comparing the two materials, we see that the coupling factors are rather comparable in the polarization stretching channel; evidently, the soft mode does not play such a profound role there. In contrast, the coupling factor $k_{15}$ is very large in $\mathrm{BaTiO}_{3}$; in fact, it is not far from unity, the maximum value consistent with stability. Indeed, this is precisely because the crystal is not far from being unstable with respect to a rotation of the polarization away from the rhombohedral axis - precisely the type of distortion that would carry it to the orthorhombic phase, from which it evolved as the temperature was reduced below the orthorhombic-rhombohedral phase transition temperature that occurs experimentally at $\sim 180 \mathrm{~K}$. The large $k_{15}$ is also strongly connected to the large difference between $\epsilon=\epsilon^{(\eta)}$ and $\epsilon^{(\sigma)}$ in Tab. VI as already discussed at the end of Sec. IV C.

The calculated value $k_{15}=0.84$ for $\mathrm{BaTiO}_{3}$ is quite respectable; it is in the range of the values of $k_{15}=0.25$ $0.80^{39}$ for the PMN-PT and PZN-PT single-crystal piezoelectrics on the rhombohedral side of the morphotropic phase boundary. Unfortunately, the fact that this large coupling occurs only at very low temperature probably makes it useless for practical applications. On the other hand, the present work suggests that if a material like $\mathrm{BaTiO}_{3}$ could somehow be stabilized in the rhombohedral phase at room temperature, it might have very promising piezoelectric properties. 


\section{SUMMARY}

In summary, we have developed a method that systematically treats the effects of perturbations associated with atomic displacements, electric fields and strains in insulating crystals, so that physical quantities expressible as second derivatives of the total energy can be computed efficiently. In the first step, six elementary tensors are computed once and for all using the methods of DFPT: the force-constant matrix, the Born charge tensor, the internal-strain tensor, and the frozen-ion dielectric, elastic, and piezoelectric tensors. The internal-displacement degrees of freedom are then eliminated to give physical low-frequency dielectric, elastic, and piezoelectric tensors, defined under boundary conditions of controlled electric field $\mathcal{E}$ and strain $\eta$. We have also shown how these can then be manipulated to obtain various related tensor properties of interest such as the free-stress dielectric tensor, the fixed- $D$ elastic and compliance tensors, and various piezoelectric tensors and electromechanical coupling factors. Such a systematic approach is especially important in polar crystals, in which the atomicdisplacement, electric-field, and strain degrees of freedom are strongly coupled in complex ways.

We have applied our approach to compute these tensor properties for two paradigmatic crystals, $\mathrm{ZnO}$ and $\mathrm{BaTiO}_{3}$, at zero temperature. These materials differ most significantly in that there is a ferroelectric soft mode that has condensed, but still remains rather low in frequency, in the latter material. The calculations are subject to several approximations, most notably the LDA itself (and its associated lattice-constant error, which has been removed by hand for the case of $\mathrm{BaTiO}_{3}$ - see Sec. III), but also the frozen-core approximation (as implemented through the use of pseudopotentials) and the neglect of zero-point fluctuations. Nevertheless, we validate the approach by finding reasonably good agreement between theory and experiment for most quantities in the case of $\mathrm{ZnO}$, despite the fact that the experiments are room-temperature ones. The largest discrepancies are for the purely electronic dielectric tensor elements $\bar{\epsilon}_{11}$ and $\bar{\epsilon}_{33}$, the shear piezoelectric coupling $e_{15}$, and to a lesser extent, derived quantities that depend on these elementary ones. In the case of $\mathrm{BaTiO}_{3}$, where low-temperature experiments on single-crystal, single-domain samples under well-defined boundary conditions are not available, our calculations provide useful predictions of the material constants. In particular, we find an encouraging value of 0.84 for the $k_{15}$ electromechanical coupling constant, and argue that this is associated with the proximity of the orthorhombic phase.

We wish to emphasize that the usefulness of the general approach advocated here transcends the particular implementation of it (here DFT/LDA, pseudopotentials, etc.). For example, similar calculations might by carried out with Hartree-Fock methods using localized orbitals ${ }^{28}$ or, eventually, using "LDA+U", dynamical mean-field the- ory, or quantum Monte Carlo methods. In this case, the six elementary tensors of Eqs. (3-8) will first need to be calculated using methods appropriate to the particular type of electronic-structure method used. However, the subsequent manipulations described in Secs. II B and II C can then be carried through in identically the same way as done here.

Finally, we note that the approach described here can be extended to include other types of perturbations, such as alchemical ones, and to the treatment of higher-order responses (e.g., anharmonic elastic constants, nonlinear dielectric responses, and electrostriction effects), providing possible directions for future developments of the method.

\section{ACKNOWLEDGMENTS}

The work was supported by ONR Grant N00014-971-0048 and by the Center for Piezoelectrics by Design under ONR Grant N00014-01-1-0365. We wish to thank K. Rabe for useful discussion, and M. Veithen for a careful reading of the manuscript.

\section{APPENDIX A: SIMULTANEOUS TREATMENT OF STRAINS AND ELECTRIC FIELDS}

The formulation of an energy functional, and the definition of response functions in terms of its second derivatives, is somewhat subtle in the case that electric fields and strains are simultaneously present. The purpose of this Appendix it to give a careful treatment of the theory in this case. Except where noted, the notation here follows that of Sec. II B in that the internal displacements $u_{m}$ are assumed to have been integrated out (i.e., internal displacements $u_{m}$ do not appear explicitly).

We begin introducing the deformation tensor $\widetilde{\eta}_{\alpha \beta}$ in the Cartesian frame via

$$
d r_{\alpha}=\widetilde{\eta}_{\alpha \beta} r_{\beta}
$$

(implied sum notation) where $d r_{\alpha}$ is the deformation of the medium from its undeformed position $r_{\alpha}$. We consider deformations taking the form of homogeneous strains and rigid rotations, so that the antisymmetry part of the deformation tensor $\widetilde{\eta}_{\alpha \beta}$ describes the rotational part, while its symmetric part is just the strain tensor

$$
\eta_{\alpha \beta}=\frac{1}{2}\left(\widetilde{\eta}_{\alpha \beta}+\widetilde{\eta}_{\beta \alpha}\right) .
$$

The improper piezoelectric tensor is defined as

$$
e_{\alpha \beta \gamma}^{\mathrm{impr}}=\frac{d P_{\alpha}}{d \widetilde{\eta}_{\beta \gamma}} .
$$

The name "improper" reflects that fact that $e^{\text {impr }}$ contains contributions that are spurious in a certain sense. ${ }^{15}$ 
For example, if we consider a pure rotation of a spontaneously polarized crystal about an axis that does not coincide with $\mathbf{P}$, then $\mathbf{d P}$ does not vanish, and consequently $e_{\alpha \beta \gamma}^{\mathrm{impr}}$ has a component that is antisymmetric under interchange of $\beta$ and $\gamma$. Similarly, if we consider a uniaxial or biaxial compression in the $x-y$ plane of a ferroelectric modeled as a lattice of discrete rigid dipoles oriented in the $z$ direction, the polarization will change even if the dipoles do not, because the polarization is defined as the dipole moment per unit volume. This, too, is an essentially spurious effect. By contrast, the "proper" piezoelectric tensor will be defined so as to vanish in either of these situations.

In the presence of a strain, it is convenient to introduce reduced or rescaled polarizations $P_{\alpha}^{\prime}$ and electric fields $\mathcal{E}_{\alpha}^{\prime}$ via

$$
P_{\alpha}^{\prime}=\left(\widetilde{\eta}^{-1}\right)_{\alpha \beta} P_{\beta}
$$

and

$$
\mathcal{E}_{\alpha}^{\prime}=\widetilde{\eta}_{\beta \alpha} \mathcal{E}_{\beta}
$$

where $P_{\alpha}^{\prime}$ and $\mathcal{E}_{\alpha}^{\prime}$ coincide with $P_{\alpha}$ and $\mathcal{E}_{\alpha}$ in the absence of strains or rotations. We then take our fundamental energy functional to be

$$
\widetilde{E}\left(\mathcal{E}^{\prime}, \widetilde{\eta}\right)=\frac{1}{\Omega_{0}}\left[E_{\mathrm{cell}}^{(0)}-\Omega \mathcal{E}^{\prime} \cdot \mathbf{P}^{\prime}\right]
$$

where $E_{\text {cell }}^{(0)}$ is the usual zero-field Kohn-Sham energy per cell ${ }^{14}$ of the occupied Bloch functions introduced earlier in Sec. II A.

Note that $\mathbf{P} \cdot \mathcal{E}=\mathbf{P}^{\prime} \cdot \mathcal{E}^{\prime}$, so that Eq. (A6) is closely related to Eq. (1). However, it is important to understand that $\mathcal{E}^{\prime}$ and $\widetilde{\eta}$ are the "natural variables" of the energy functional (A6), so that subsequent partial derivatives are defined in terms of this pair of variables. For example, the proper piezoelectric tensor is now given by

$$
e_{\alpha \beta \gamma}=-\frac{\partial^{2} \widetilde{E}}{\partial \mathcal{E}_{\alpha}^{\prime} \partial \widetilde{\eta}_{\beta \gamma}}
$$

or

$$
e_{\alpha \beta \gamma}=\frac{1}{\Omega_{0}} \frac{\partial\left(\Omega P_{\alpha}^{\prime}\right)}{\partial \widetilde{\eta}_{\beta \gamma}}
$$

We also emphasize that $\mathcal{E}^{\prime}$ is, in many ways, a more natural variable than $\mathcal{E}$ from the experimental point of view. For example, if one controlls the voltage $V$ across a film of $M$ atomic layers between conducting capacitor plates and observes the resulting strain, one is actually controlling $\mathcal{E}^{\prime}=e V / M c_{0}=\mathcal{E} c / c_{0}$, not $\mathcal{E}$, where $c_{0}$ and $c$ are the zero-field and finite-field lattice constants, respectively, in the normal direction.

From Eqs. (A3) and (A8) it follows that the improper and proper piezoelectric tensors are related by ${ }^{15}$

$$
e_{\alpha \beta \gamma}=e_{\alpha \beta \gamma}^{\mathrm{impr}}-\delta_{\beta \gamma} P_{\alpha}+\delta_{\alpha \beta} P_{\gamma}
$$

It is then easy to show that the proper tensor $e_{\alpha \beta \gamma}$ is symmetric under interchange of indices $\beta$ and $\gamma$, so that the Voigt notation can be restored. This is to be expected because the reduced quantity $\mathbf{P}^{\prime}$ is invariant under a rigid rotation of the crystal, a fact that follows trivially from Eq. (A4). That is, $\mathbf{P}^{\prime}$, expressed as a function of the six symmetrized strain variables (Eq. (A2)) and the three rotational variables, is actually independent of the rotational ones. Indeed, a rigid rotation of the entire system, material plus external field $\mathcal{E}$, leaves both $\mathcal{E}^{\prime}$ and $\mathbf{P}^{\prime}$ individually unchanged. It is then natural to discard the rotational variables and recast the symmetric strain variables in Voigt notation. We then regard the energy functional of Eq. (A6) to be a functional $\widetilde{E}\left(\mathcal{E}^{\prime}, \eta\right)$ of the fundamental variables of rescaled $\mathcal{E}_{\alpha}^{\prime}$ and Voigt $\eta_{j}$, and the proper piezoelectric tensor may be written as

$$
e_{\alpha j}=-\frac{\partial^{2} \widetilde{E}}{\partial \mathcal{E}_{\alpha}^{\prime} \partial \eta_{j}}=\frac{1}{\Omega_{0}} \frac{\partial\left(\Omega P_{\alpha}^{\prime}\right)}{\partial \eta_{j}} .
$$

Restoring the explicit dependence on internal displacements $u_{m}$, Eq. (A6) becomes

$$
E\left(u, \mathcal{E}^{\prime}, \eta\right)=\frac{1}{\Omega_{0}}\left[E_{\text {cell }}^{(0)}-\Omega \mathcal{E}^{\prime} \cdot \mathbf{P}^{\prime}\right],
$$

where $\widetilde{E}\left(\mathcal{E}^{\prime}, \eta\right)$ of Eq. (A6) corresponds to the minimum of (A11) over all possible displacements $u_{m}$. While this equation is numerically equal to Eq. (1), it is critical to recall that it is written in terms of different arguments.

Strictly speaking, this notation should have been introduced at the very beginning of Sec. II A, and every equation throughout the paper, starting with Eq. (1), should have $\mathcal{E}$ replaced by $\mathcal{E}^{\prime}$ and $\mathbf{P}$ by $\mathbf{P}^{\prime}$. For example, Eqs. (8) and Eq. (26) should be replaced by Eqs. (A12) and

$$
\bar{e}_{\alpha j}=-\frac{\partial^{2} E}{\partial \mathcal{E}_{\alpha}^{\prime} \partial \eta_{j}},
$$

respectively, and similarly for all other equations. However, for the purposes of clarity of presentation, it was decided to avoid use of this clumsy notation in the main part of the paper.

Finally, we note that the reduced quantities $\mathbf{P}^{\prime}$ and $\mathcal{E}^{\prime}$ are also rather natural physical variables from the point of view of computational implementation. Indeed, these two quantities can further be expressed in terms of fully reduced quantities $p_{\mu}$ and $\varepsilon_{\mu}$ via

$$
\mathbf{P}^{\prime}=\frac{e}{\Omega} p_{\mu} \mathbf{a}_{\mu}^{(0)}
$$

and

$$
\varepsilon_{\mu}=e \mathcal{E}^{\prime} \cdot \mathbf{a}_{\mu}^{(0)}
$$

so that $\mathbf{P}=(e / \Omega) p_{\mu} \mathbf{a}_{\mu}$ and $\varepsilon_{\mu}=e \mathcal{E} \cdot \mathbf{a}_{\mu}$. In these equations, $\mathbf{a}_{\mu}^{(0)}$ is the $\mu$ 'th undeformed primitive real-space 
lattice vector, $\mathbf{a}_{\mu}$ is the corresponding deformed lattice vector, and $\Omega=\mathbf{a}_{1} \cdot \mathbf{a}_{2} \times \mathbf{a}_{3}$ is the deformed cell volume. Note that $\mathbf{P} \cdot \mathcal{E}=\mathbf{P}^{\prime} \cdot \mathcal{E}^{\prime}=\Omega^{-1} p_{\mu} \varepsilon_{\mu}$ and

$$
e_{\alpha j}=\frac{e}{\Omega_{0}} a_{\mu, \alpha} \frac{\partial p_{\mu}}{\partial \eta_{j}} .
$$

The fully reduced polarization $p_{\mu}$ has a simple interpretation in terms of the fractional positions of the charges in the unit cell; for example, the contribution to it coming from filled band is just $-1 / 2 \pi$ times the Berry phase of that band, as can be seen by comparing with Eq. (10) of Ref. 15. Similarly, $\varepsilon_{\mu}$ is just $e$ times the electrostatic potential drop across the unit cell in direction $\mathbf{a}_{\mu}$.

The computational implementation of DFPT is done quite naturally in terms of these reduced quantities, $7,10,19$ and as a result, DFPT automatically yields the proper piezoelectric tensor. ${ }^{10}$ This can be a source of confusion when comparing the DFPT results with those of finite-difference calculations. In the latter approach, the polarization is obtained directly from ground-state DFT calculations, ${ }^{6}$ and piezoelectric tensor elements are obtained by numerical differentiation using sufficiently small strains about the reference structure. This procedure yields the improper tensor, however, and Eq. (A9) must be applied to compare such results with the DFPT ones. $^{10}$

${ }^{1}$ P. Hohenberg and W. Kohn, Phys. Rev. 136, B864(1964); W. Kohn and L. J. Sham, ibid. 140, A1133 (1965).

${ }^{2}$ X. Gonze, Phys. Rev. A 52, 1086 (1995); 52, 1096 (1995); 55, 10337 (1997); X. Gonze and C. Lee, ibid. 55, 10355 (1997).

${ }^{3}$ S. Baroni, S. de Gironcoli, A. Dal Corso, and P. Giannozzi, Rev. Mod. Phys. 73, 515 (2001)

${ }^{4}$ P. Giannozzi, S. de Gironcoli, P. Pavone, and S. Baroni, Phys. Rev. B 437231 (1991); S. Baroni, P. Giannozzi, and A. Testa, Phys. Rev. Lett. 58, 1861 (1987).

${ }^{5}$ S. de Gironcoli, S. Baroni, and R. Resta, Phys. Rev. Lett. 62, 2853 (1989).

${ }^{6}$ R. D. King-Smith and D. Vanderbilt, Phys. Rev. B 47, 1651 (1993).

${ }^{7}$ ABINIT is a common project of the Université Catholique de Louvain, Corning Incorporated, and other contributors (http://www.abinit.org). X. Gonze, J.-M. Beuken, R. Caracas, F. Detraux, M. Fuchs, G.-M. Rignanese, L. Sindic, M. Verstraete, G. Zerah, F. Jollet, M. Torrent, A. Roy, M. Mikami, Ph. Ghosez, J.-Y. Raty, and D. C. Allan, Comput. Mater. Sci. 25, 478 (2002).

${ }^{8}$ S. Baroni, A. Dal Corso, S. de Gironcoli, P. Giannozzi, C. Cavazzoni, G. Ballabio, S. Scandolo, G. Chiarotti, P. Focher, A. Pasquarello, K. Laasonen, A. Trave, R. Car, N. Marzari, A. Kokalj, http://www.pwscf.org.

${ }^{9}$ G. Kresse and J. Hafner, Phys. Rev. B 47, R558 (1993);
G. Kresse and J. Furthmüller, ibid. 54, 11169 (1996). See also http://cms.mpi.univie.ac.at/vasp.

${ }^{10}$ D. R. Hamann, D. Vanderbilt, K. Rabe, and X. Wu, submitted to Phys. Rev. B.

${ }^{11}$ R. W. Nunes and X. Gonze, Phys. Rev. B 63, 155107 (2001).

12 I. Souza, J. Íñiguez, D. Vanderbilt, Phys. Rev. Lett. 89, 117602 (2002).

${ }^{13}$ P. Umari and A. Pasquarello, Phys. Rev. Lett. 89, 157602 (2002).

14 That is, the electrostatic part of $E_{\text {cell }}^{(0)}$ is defined to be $\left(\epsilon_{0} / 2\right) \int_{\text {cell }} d \mathbf{r} \mathcal{E}_{\text {loc }}^{2}(\mathbf{r})$, where the local field $\mathcal{E}_{\text {loc }}(\mathbf{r})$ is the difference between the microscopic field $\mathcal{E}(\mathbf{r})$ and its unit-cell average $\mathcal{E}$.

${ }^{15}$ D. Vanderbilt, J. Phys. Chem. Solids 61, 147 (2000).

${ }^{16}$ A. Ballato, IEEE Trans. Ultrason., Ferroelec., Freq. Contr., 42, 916 (1995).

17 J.F. Nye, Physical properties of crystals (Oxford U.P., Oxford 1985).

18 ANSI/IEEE Standard on Piezoelectricity (ANSI/IEEE Std 176-1987), IEEE Transactions on Sonics and Ultrasonics SU-31, No. 2, 1984.

19 The capabilities to do these calculations are now incorporated in the latest public release of the ABINIT software package. ${ }^{7}$ Building on the implementation of the strain derivatives for computation of bare elastic, piezoelectric, and internal-strain tensors by Hamann et al. ${ }^{10}$ we modified ABINIT (specifically, the ANADDB module) to provide the functionality of computing the secondary response tensors as described here.

${ }^{20}$ In the case of $Z$ and $e$, the wavefunction derivatives with respect to wavevector are also needed (see Ref. 2).

${ }^{21}$ N. Troullier and J. L. Martins, Phys. Rev. B 43, 1993 (1991).

22 N. Hill and U. Waghmare, Phys. rev. B 62, 8802 (2000).

${ }^{23}$ D. M. Ceperley, Phys. Rev. B 18, 3126 (1978); D. M. Ceperley and B. J. Alder, Phys. Rev. Lett. 45, 566 (1980).

24 J. P. Perdew and Y. Wang, Phys. Rev. B 45, 13244 (1992).

${ }^{25}$ W. Zhong and D. Vanderbilt, Phys. Rev. B 52, 6301 (1995)

${ }^{26}$ D. Berlincourt and H. Jaffe, Phys. Rev. 111, 143 (1958).

${ }^{27}$ M. Zgonik, P. Bernasconi, M. Duelli, R. Schlesser, P. Günter, M. H. Garrett, D. Rytz, Y. Zhu, and X. Wu, Phys. Rev. B 50, 5941 (1994).

${ }^{28}$ M. Catti, Y. Noel, R. Dovesi, J. Phys. Chem. Solids 64 2183, (2003).

${ }^{29}$ O. Madelung, M. Schultz, and H. Weiss, Numerical Data and Functional and Relationships in Science and Technology (Springer-Verlag, Berlin, 1982), Vol. 17.

${ }^{30}$ A. W. Hewat, Ferroelectrics, 6, 215 (1974).

31 T. C. Damen, S. P. S. Porto, and B. Tell, Phys. Rev. 142, 570 (1966).

${ }^{32}$ C. A. Arguello, D. L. Rousseau, and S. P. S. Porto, Phys. Rev. 181, 1351 (1969).

${ }^{33}$ P. Ghosez, Ph.D. thesis, Universite Catholique de Louvain, 1997.

${ }^{34}$ N. Ashkenov, B. N. Mbenkum, C. Bundesmann, V. Riede, M. Lorenz, D. Spemann, E. M. Kaidashev, A. Kasic, M. Schubert, M. Grundmann, G. Wanger, H. Neumann, V. Darakchieva, H. Arwin, and B. Monema, J. Appl. Phys. 93, 126 (2003). 
${ }^{35}$ B. Wang and C. Sun, Appl. Opt. 40, 672 (2001).

${ }^{36}$ Landolt-Börnstein, O. Madelung (Ed.), New Series, Group III: Solid State Physics, Low Frequency Properties of Dielectric Crystals: Piezoelectric, Pyroelectric and Related Constants, vol. 29b, Springer, Berlin, 1993.

${ }^{37}$ A. Dal Corso, M. Posternak, R. Resta, and A. Baldereschi, Phys. Rev. B 50, 10715 (1994).

${ }^{38}$ F. Bernardini, V. Fiorentini, and D. Vanderbilt, Phys. Rev. B 56, 10024 (1997).

${ }^{39}$ H. Cao, V. H. Schmidt, R. Zhang, W. Cao and H. Luo, J. appl. Phys. 96, 549 (2004); R. Zhang, B. Jiang, W. Jiang and W. Cao, Mater. Lett. 57, 1305(2003). 